\section{TRISTETRAPROLIN POST-TRANSCRIPTIONALLY REGULATES TISSUE FACTOR EXPRESSION IN PRIMARY HUMAN AND MURINE MACROPHAGES}

doi:10.1136/heartinl-2012-302951.001

Presenter: Bilal Iqbal

'MB labal, ${ }^{2} J E$ Dean, 'N Burke, ${ }^{2} \mathrm{~A}$ Clark, 'M Johns, 'DO Haskard. 'Vascular Sciences Section, National Heart and Lung Institute, Hammersmith Hospital, Imperial College London; ${ }^{2}$ Kennedy Institute, University of Oxford

Background Although monocyte-derived tissue factor (TF) plays critical roles in atherothrombosis, little is known about its posttranscriptional regulation. Tristetraprolin (TTP) binds the 3'UTR of target mRNAs and promotes their degradation, with its function being negatively regulated by p38 MAPK. Whether TTP posttranscriptionally regulates TF is unknown.

Methods We used human monocytes and bone marrow derived macrophages from TTP $/+$ and TTP-/- mice. Procoagulant activity was determined using a clot turbimetric assay. mRNA decay was determined following transcriptional arrest using actinomycin D. TTP knockdown was achieved using siRNA transfection. RNA and protein interaction was determined using ribonucleoprotein (RNP) immunoprecipitation and RNA biotin pulldown assays.

Results p38 inhibition with SB203580 and SB202190 (1 $1 \mu \mathrm{MM})$ reduced procoagulant activity, TF $\mathrm{mRNA}$ and protein expression in human macrophages $(p<0.05)$. p38 inhibition reduced TF mRNA stability in both human and murine macrophages $(p<0.05)$. TTP knockdown increased TF expression in human macrophages $(p<0.05)$. Both TF mRNA and protein expression were significantly increased in TTP-/- versus TTP-/- macrophages $(p<0.05)$. Moreover TF mRNA decay was reduced in TTP-/- macrophages and p38 inhibition had no effect on this $(p<0.01)$. RNP immunoprecipitation demonstrated TTP and TF mRNA interaction. Furthermore, a more specific interaction with TTP and TF 3'UTR was confirmed using RNA biotin pulldown techniques.

Conclusions These data provide evidence, for the first time, that p38 and TTP post-transcriptionally regulate TF expression in macrophages. A better understanding of the post-transcriptional regulation of TF expression will provide novel insights into the interface between inflammation and thrombosis in vascular biology, and holds therapeutic potential.

NANOPARTICLES FOR SIMULTANEOUS CELL TRACKING \& MICRORNA DELIVERY

Presenter: Renata Gomes

doi:10.1136/heartjnl-2012-302951.002

1,2,3,4RSM Gomes, ${ }^{1,2} \mathrm{R}$ Neves, ${ }^{4}$ Cochlin, LE, ${ }^{1,2}$ AF Lima, ${ }^{2} \mathrm{R}$ Carvalho, ${ }^{5} \mathrm{P}$ Korpisalo, ${ }^{5} \mathrm{G}$ Dragneva, ${ }^{2} \mathrm{APaiva},{ }^{5} \mathrm{~S}$ Ylä-Herttuala, ${ }^{4} \mathrm{~K}$ Clarke, ${ }^{4} \mathrm{CACarr},{ }^{1,2} \mathrm{~L}$ Ferreira. ${ }^{1}$ Biomaterials $\&$ Stem Cells Based Therapeutics Lab, Biocant, Cantanhede, Portugal; ' 2 Centre of Neurosciences and Cell Biology, University of Coimbra, Portugal; ${ }^{3}$ Cardiovascular Biology \& Medicine, Rayne institute, University College London, UK; ${ }^{4}$ Cardiac Metabolism Research Group, Department of Physiology, Anatomy \& Genetics, Oxford University, UK; ${ }^{5}$ Gene Therapy \& Angiogenesis group, University of Eastern Finland, Finland

Magnetic resonance imaging (MRI) is a powerful non-invasive imaging platform which, in combination with contrast agents, can be used to track stem cell fate after transplantation. Here, we developed a novel formulation of nanoparticles (NPs), based on poly(lactic-co-glycolic acid) (PLGA) which is a biocompatible polymer approved for human use. PLGA NPs are bioluminescent and contain fluorine, enabling cell tracking using optical imaging or 19F MRI, and can be used as vectors for microRNA (miR) delivery.
The PLGA NPs (average diameter 170nm, positive $(7.0 \pm 1.7$ $\mathrm{mV}$ ) zeta potential) successfully labelled HUVECs $(63.18 \pm 0.96 \%)$ and mononuclear cells (MNCs; $80.75 \pm 1.21 \%$ ) with only $10 \%$ of cells dying after $24 \mathrm{~h}$ of NP exposure. Labelled MNCs, administered to the infarcted rat heart, could be detected in vivo using 19F MRI

HUVECs were incubated with miRNA-complexed NPs to deliver pro-survival and pro-angiogenic miRs (miR-132, miR-424 and antagomir-92a (amiR-92a)) and cultured on matrigel to assess their capacity to form vascular networks. Cells transfected with miR-132 NPs and miR-424 NPs yielded vascular networks with greater tube length and more branching points than those transfected with amiR-92a NPs. Furthermore, NPs were more efficient at transfection than the commercially available reagent, SIPORT.

Endothelial cells labelled with miR-132 NPs were administered to rodent models of hind limb ischemia. Labelled cells could be tracked in vivo using 19F MRI, improved cell survival and induced neo-angiogenesis (measured using ultrasound and histology).

This work shows for the first time a NP formulation that can be used both to track cells and successfully deliver miRs.

\section{TISSUE TRAIL DRIVES PULMONARY VASCULAR REMODELING AND ITS INHIBITION REVERSES EXPERIMENTAL PULMONARY ARTERIAL HYPERTENSION}

doi:10.1136/heartjnl-2012-302951.003

\section{Presenter: Abdul Hameed}

AG Hameed, ND Arnold, J Chamberlain, J Pickworth, CMH Newman, ${ }^{2}$ DC Crossman, SE Francis \& A Lawrie. 'Department of Cardiovascular Science, University of

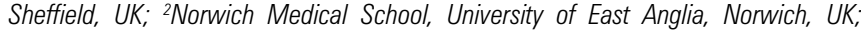
${ }^{3}$ Norwich and East

Background and aims Pulmonary arterial hypertension (PAH) is a fatal disease characterised by progressive narrowing of pulmonary arterioles, driven by aberrant cellular proliferation. Identifying key pathways in disease pathogenesis is required for the development of new-targeted therapies.

We have previously reported Tumour Necrosis Factor (TNF)Related Apoptosis-Inducing Ligand (TRAIL) immunoreactivity within pulmonary vascular lesions from patients with idiopathic PAH and animal models. Since TRAIL induces endothelial cell apoptosis and smooth muscle cell proliferation, we hypothesised that TRAIL is an important driver of disease in PAH.

Methods Using the Paigen diet-fed ApoE-/- murine model, we first tested whether genetic deletion (ApoE-/-/TRAIL-/-) and/or antiTRAIL antibody treatment could modulate disease progression. Bone marrow transplantation (BMT) from ApoE-/- into sub lethally irradiated ApoE-/-/TRAIL-/- mice, and vice versa was performed. Phenotyping included cardiac catheterisation (Right Ventricular Systolic Pressure) and immunohistological analyses of excised lung tissue.

Results ApoE-/-/TRAIL-/- mice were protected from developing PAH (RVSP $28 \mathrm{mmHg}$ v. $50 \mathrm{mmHg}, \mathrm{P}<0.001, \mathrm{n}=6$ ). Anti-TRAIL antibody treatment of ApoE-/- mice with established disease reversed PAH (RVSP $27 \mathrm{mmHg}$ v. $88 \mathrm{mmHg}, \mathrm{P}<0.05, \mathrm{n}=4$ ). Blocking TRAIL significantly decreased cellular proliferation and increased apoptosis within pulmonary arterioles.

In chimaeras, only mice with expression of TRAIL restricted to tissue developed significant PAH (Mean RVSP $47 \mathrm{mmHg}$ v. $26 \mathrm{mmHg}$ $\mathrm{p}<0.01, \mathrm{n}=4-6)$. Mice with TRAIL only expressed by bone marrow derived cells showed no significant signs of $\mathrm{PAH}$.

Conclusions Our studies are the first to determine the importance of TRAIL in the pathogenesis of PAH and demonstrate its potential for translation into a novel therapeutic target. 